\title{
Enterocolitis in the evolution of an early diagnosed Hirschsprung disease - case report
}

\author{
Georgiana-Emmanuela Gîlcă ${ }^{1}$, Carmen lulia Ciongradi ${ }^{\star 1,2}$ \\ 1 "Grigore T. Popa" University of Medicine and Pharmacy, lasi, Romania, ${ }^{2} 2^{\text {nd }}$ Pediatric Surgery Clinic, \\ "Sf. Maria" Emergency Hospital for Children, Iasi, Romania
}

\begin{abstract}
Hirschsprung disease associated enterocolitis (HAEC) represents a recognized complication with multifactorial etiology, remaining a leading cause of morbidity and mortality among infants with Hirschsprung disease. This report describes the case of an infant boy with a history of delay in meconium passage and bilious vomiting at 2 days of age, presenting at a subsequent admittance with presumptive suboclusive syndrome, which turns out to be after rigorous investigations a Hirschsprung disease complicated with enterocolitis. The case highlights the importance of prompt recognition and energetic intervention for HAEC, which together with choosing the appropriate surgical approach leads to a beneficial outcome in patient's evolution.
\end{abstract}

Keywords: HAEC, LATEP, outcome

\section{Introduction}

Hirschsprung disease (HD) appears as a result of anomalies in enteric nervous system development and neural cell crest migration during embryogenesis, determining aganglionosis in a variable portion of the gut, usually affecting the rectum and distal colon [1-4]. The clinical picture of HD can be variable, including directly the ones caused by complications' emergence, among which enterocolitis is the most redoubtable, having a multifactorial pathogenesis and possibility to arise both in the preoperative period and after surgical management of HD, even independently of the medical management or surgical approach chosen [5].

Received: August 2014; Accepted after review: September 2014; Published: September 2014

${ }^{*}$ Corresponding author: Carmen lulia Ciongradi, MD, $\mathrm{PhD}, 2^{\text {nd }}$ Pediatric Surgery Clinic, "Sf. Maria" Emergency Hospital for Children, Iasi, "Grigore T. Popa" University of Medicine and Pharmacy, lasi, Romania. Email: iuliaciongradi@yahoo.com
Clinically, HAEC can overlap with other conditions such as gastroenteritis or obstructive bowel syndrome, possibly leading to misdiagnosis [6].

Taking into account this argument and also the impact of HAEC on patient's outcome, efforts have been made in order to clearly assess a standard definition and estimate the incidence of HAEC and also to find possible prophylaxis methods such as colonic irrigation and probiotic administration $[7,8]$.

\section{Case presentation}

An 18 day-old newborn was admitted in the Pediatric Surgery Clinic for marked abdominal distention and bilious vomiting beginning 1 day before the admission. The infant presented the following features at the clinical examination: influenced general status, pale dehydrated tegument, distended abdomen with palpable dilated loops of colon. Rectal examination revealed an empty rectal vault. 
The infant was previously referred to the same clinic at the age of 2 days for delay in meconium passing. It is noted that the child was delivered by a cesarean section, as a consequence of transverse presentation, after a gestation period without pathological findings.

Paraclinical investigations included abdominal ultrasound which identified high quantity of peritoneal fluid, abdominal plain Xray revealing important aeric distention in the upper two-thirds of the abdomen, air-fluid levels in the medium abdominal third, and opacity of the pelvis.

Biological findings highlighted leukocytosis with polynucleosis, thrombocytosis, normocytic anemia, hypoalbuminemia and complex metabolic imbalance (metabolic acidosis with respiratory alkalosis), leading to the stage diagnosis of sepsis through enterocolitis in the context of a presumable Hirschsprung disease. Therefore, a stool sample was taken for coproculture, subsequently identifying $C$. difficile as pathogenic agent. In order to overcome the metabolic imbalance and resolve sepsis, antibacterial chemotherapy (broad-spectrum at first, and C. difficile- targeted after coproculture results) and intravenous fluid resuscitation were administered, joined with digestive repose, placement of a nasogastric tube and proper nursing (rectal irrigation) for colon evacuation.

A gradual improvement of the patient's status was noticed and additional investigations were performed, in order to acquire a better evidence of sepsis' cause.

Contrast enema revealed important gas distension of the colon, with a narrowing of the distal colon at less than half the normal diameter, with proximal dilation, the presence of a transition zone between sigmoid and descendent colon; important reduction of the rectum caliber, with filamentous ending, typical aspect for the Hirschsprung disease, with rectosigmoid aganglionosis (Figure 1).

A suction rectal biopsy for a histological confirmation of the diagnosis was not performed, due to technical limitations, but the clinical features, patient history and imagistic evidence were highly suggestive in order to support the diagnosis.

Echocardiography and genetic testing (karyotyping) were performed in order to rule out potential associated anomalies.

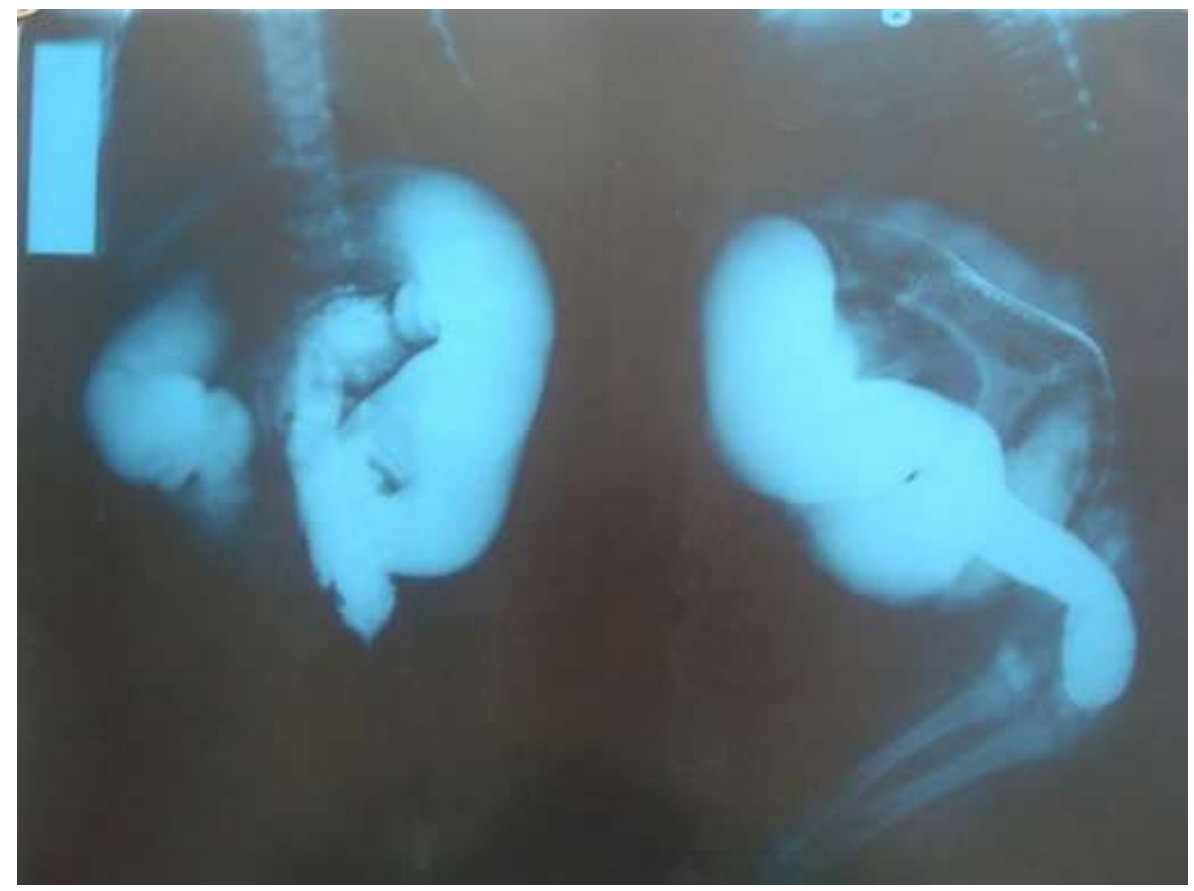

Fig. 1. Contrast enema revealing narrowed rectosigmoidian region and highlighting the transition zone 


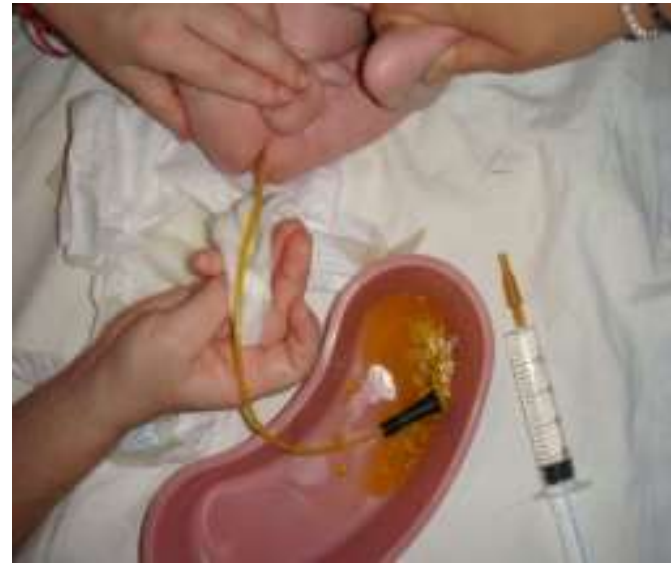

Fig. 2. Colon washout in order to assess abdominal decompression and content evacuation

Given the favorable evolution of the acute episode and the patient's age and status, the surgical intervention was temporized, with assuring a proper prevention of complications and providing daily colon washout (Figure 2) at home until 8 months of age, when a laparoscopic assisted transanal pull-through (LATEP) with removal of the aganglionic colon segment and coloanal anastomosis using normally innervated colon was performed (Figures 3-6).

Along with it, biopsies of the rectum and of the colon at various levels were performed in order to histologically confirm the diagnosis and to accurately identify the level at which normal innervation of the colon is found.

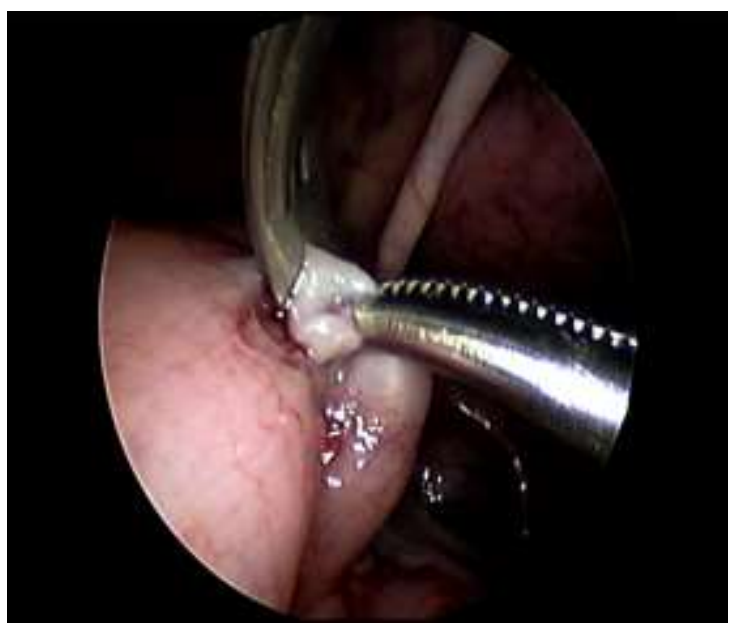

Fig. 3. LATEP. Identifying the aganglionic segment and performing biopsy

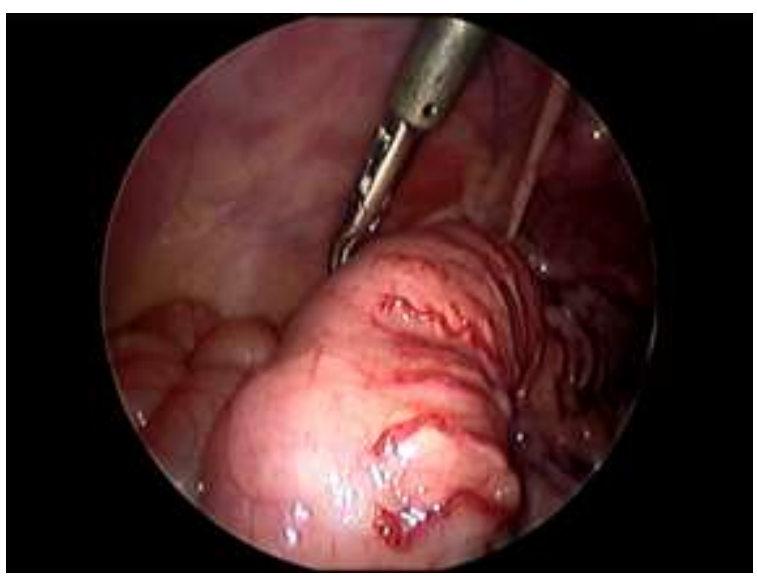

Fig. 4. LATEP. Identifying normal innervated colon and obtaining biopsy for confirmation

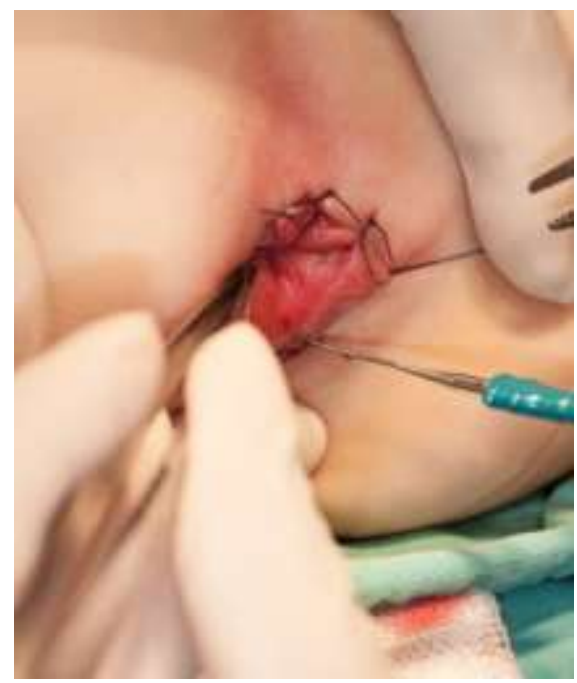

Fig. 5. LATEP. Extramucous rectal resection

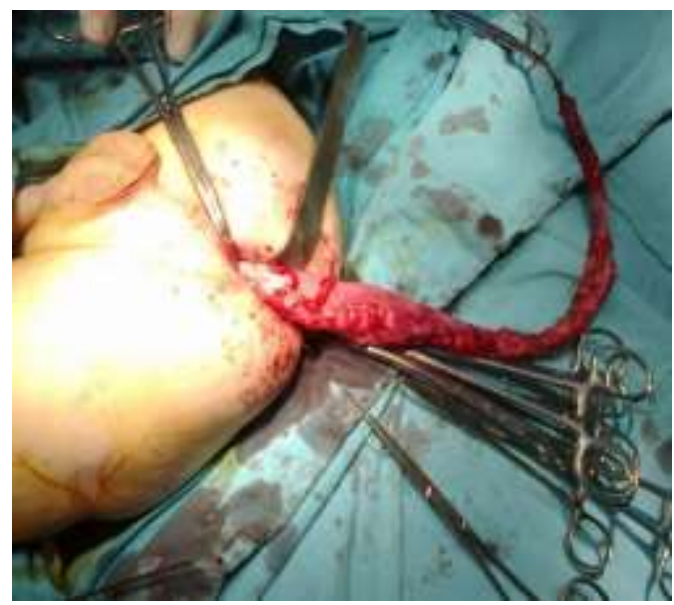

Fig. 6. LATEP. Visualisation of colon after pull through, highlighting the transition zone 
The postoperative evolution was favorable with neither early, nor late complications during next 2 years follow-up period, mentioning that for the consequent period it is essential to monitor the degree of continence and the eventuality of soiling.

\section{Discussions}

$\mathrm{HD}$ is one of the most common causes of intestinal obstruction in newborn, being caused by the agangliosis of the bowel, from the internal anal sphincter to a variable distance proximally, most frequently affecting the rectosigmoid region. A delay in meconium passage should raise awareness towards investigating the possibility of $\mathrm{HD}$ in a newborn, as in our case, in order to avoid complications such as intestinal obstruction, sepsis, intestinal perforation and recurrent enterocolitis, last of which represents an important complication because of its impact on patient's prognosis, having a high morbidity and mortality rate.

Moreover, the clinical presentation can be directly influenced by the complications arising in HD's evolution, among which enterocolitis is considered to be multifactorial, mainly based on the connection between the agangliosis characterizing Hirschsprung disease, defective intestinal barrier and influence of altered commensal microflora $[7,8]$.

The definitive treatment for HD is surgical and great progress has been made to surgical approach, meaning one-stage procedures and minimally invasive techniques, allowing decreasing the age for performing the intervention. However, there are various situations that contraindicate the intervention such as: enterocolitis, proximal bowel overdistension, precarious nutritional status.

Various complications (e.g., enterocolitis) can delay the intervention, but there have been progresses in their prophylaxis. Hence, the use of colon irrigations, anal dilations and use of probiotics are some of the methods applied, although there is controversy regarding the efficiency of probiotics' use [7, 9].
The presented case is individualized by an early occurrence of enterocolitis complicating an early diagnosed Hirschsprung disease, complication which could have signed a negative prognosis on patient's outcome. However, family's compliance regarding daily colon evacuation and also the presence of a rectosigmoid agangliosis, not of a pancolic involvement, contributed to a favorable evolution.

Moreover, laparoscopic assisted pullthrough for the definitive treatment is known to be associated with faster recovery period and fewer perioperative complications, as it reduces the adhesion formation and offers the possibility of a better mobilization and dissection of the aganglionic colon [11, 12].

Another distinctive feature of our case is that HAEC arose in a low risk patient with HD considering that main risk factors include family history, Trisomy-21, long-segment disease [5].

Regarding the early appearance of preoperative HAEC, we should stress that it represents an important factor in relation to the functional outcome after surgery for HD, that is why thorough and long term monitoring of the patient regarding bowel function and soiling is essential [13].

\section{Conclusions}

Early diagnosis of $\mathrm{HD}$ and preventing the disease's complications along with a prompt recognition of HAEC allows early intervention and a potential reduction in disease severity and mortality $[13,14]$.

Efficient colon washout program, together with prompt surgical expertise and multidisciplinary support are viewed as key features in prevention of HAEC and increasing the possibility of a good long-term outcome for the patient [6].

Regarding the surgical procedure, choosing the appropriate time and technique, including opting for a less invasive approach is a key factor for a good long-term functional outcome $[2,11,15$, and 16]. 


\section{References}

1. Gariepy CE. Intestinal motility disorders and development of the enteric nervous system. Pediatr Res 2001; 49:605-613.

2. Haricharan RN, Georgeson KE. Hirschsprung disease. Semin Pediatr Surg 2008; 17:266-275.

3. Iwashita T, Kruger GM, Pardal R, Morrison SJ. Hirschsprung disease is linked to defects in neural crest stem cell function. Science 2003; 301:972-976.

4. Theocharatos S, Kenny SE. Hirschsprung's disease: Current management and prospects for transplantation of enteric nervous system progenitor cells. Early Hum Dev 2008; 84(12):801-804.

5. Frykman PK. Hirschsprung - associated enterocolitis: prevention and therapy. Semin Pediatr Surg 2012; 21(4):328-335.

6. Austin KM. The pathogenesis of Hirschsprung's disease-associated enterocolitis. Semin Pediatr Surg 2012; 21(4):319-327.

7. El-Sawaf $M$, Siddiqui $S$, Mahmoud $M$, Drongowski R, Teitelbaum DH. Probiotic prophylaxis after pullthrough for Hirschsprung disease to reduce incidence of enterocolitis: a prospective, randomized, double-blind, placebo-controlled, multicenter trial. J Pediatr 2013; 48(1):111-117.

8. Pastor AC, Osman F, Teitelbaum DH, Caty MG, Langer JC. Development of a standardized definition for Hirschsprung associated enterocolitis: a Delphi analysis. J Pediatr Surg 2009; 44: 251-256.

9. Wang JS, Lee HC, Huang FY, Chang PY, Sheu JC. Unexpected mortality in pediatric patients with postoperative Hirschsprung's disease. Pediatr Surg Int 2004; 20:525-528.

10. Vieten D, Spicer R. Enterocolitis complicating Hirschsprung's disease. Semin Pediatr Surg 2004; 13(4):263-272.

11. van de Ven TJ. Transanal endorectal pullthrough for classic segment Hirschsprung's disease: With or without laparoscopic mobilization of the rectosigmoid? J Pediatr Surg 2013; 48:1914-1918.

12. Butler Tjaden NE, Trainor PA. The developmental etiology and pathogenesis of Hirschsprung disease. Transl Res 2013; 162(1):1-15.

13. Murthi GVS, Raine PAM. Preoperative enterocolitis is associated with poorer longterm bowel function after Soave-Boley endorectal pull-through for Hirschsprung's disease. J Pediatr Surg 2003; 38:69-72.

14. de la Torre L, Langer JC. Transanal endorectal pull-through for Hirschsprung disease: technique, controversies, pearls, pitfalls, and an organized approach to the management of postoperative obstructive symptoms. Semin Pediatr Surg 2010; 19:96-106.

15. Langer JC. Laparoscopic and transanal pullthrough for Hirschsprung Disease. Semin Pediatr Surg 2012; 21:283-290.

16. Granström AL, Husberg $B$, Nordenskjöld A, Svensson PJ, Wester T. Laparoscopic-assisted pull-through for Hirschsprung's disease, a prospective repeated evaluation of functional outcome. J Pediatr Surg 2013; 48:2536-2539. 